\title{
Reliability Estimation for Zero-Failure Data Based on Confidence Limit Analysis Method
}

\author{
Haiyang $\mathrm{Li} \mathbb{C}^{1,2}$ and Zeyu Zheng ${ }^{1,2}$ \\ ${ }^{1}$ Shenyang Institute of Automation, Chinese Academy of Sciences, Shenyang 110016, China \\ ${ }^{2}$ Institutes for Robotics and Intelligent Manufacturing, Chinese Academy of Sciences, Shenyang 110169, China \\ Correspondence should be addressed to Haiyang Li; lihaiyang@sia.cn
}

Received 28 May 2020; Revised 27 August 2020; Accepted 20 October 2020; Published 4 November 2020

Academic Editor: Huchang C. Liao

Copyright $\odot 2020$ Haiyang Li and Zeyu Zheng. This is an open access article distributed under the Creative Commons Attribution License, which permits unrestricted use, distribution, and reproduction in any medium, provided the original work is properly cited.

\begin{abstract}
Due to the improvement of the quality of industrial products, zero-failure data often occurs during the reliability life test or in the service environment, and such problems cannot be handled using traditional reliability estimation methods. Regarding the processing and analysis of zero-failure data, the confidence limit assessment methods were proposed by some researchers. Based on the existing research, a confidence limit method set (CLMS) is established in the Weibull distribution for reliability estimation of zero-failure data. The method set includes the unilateral confidence limit method and optimal confidence limit method, so that almost all existing grouping types of zero-failure data can be quickly evaluated, and multiple methods can be used in parallel to deal with the same problem. The effectiveness and high efficiency of the CLMS combined with numerical simulation examples have been verified, and the possibility of analyzing multiple groups of zero-failure data with a confidence limit method suitable for processing single group of zero-failure data is expanded. Finally, the actual effect of the method set is verified by the single group of zero-failure data of rolling bearings and the multiple groups of zero-failure data of torque motors. The results of the example evaluation show that the CLMS has obvious advantages in practical engineering applications.
\end{abstract}

\section{Introduction}

For high-reliability industrial products, it takes quite a long time to test their samples failure data. Some of the industrial products with complicated structure and expensive cost are difficult to carry out damage tests because of economic reasons [1]. Research on reliability evaluation and life prediction methods of such products can often only rely on zero-failure data with limited capacity. The traditional reliability assessment method involves less processing of zerofailure data. Therefore, the theory of reliability evaluation under the condition of zero-failure data has been developed [2].

Estimating accurate sample reliability based on zerofailure data is challenging [3]. The reliability analysis of zerofailure data is a new question encountered in recent years with the improvement of product quality. The research work is not only of theoretical significance, but also of practical application value. Since the publication of research results by
Martz and Waller [4], the research on zero-failure data has been studied for more than 40 years, which has gradually attracted the attention of related researchers [5]. Chen proposed the reliability assessment confidence limit method under the condition of zero-failure data in the 1990s and then proposed that the lower confidence limits of the reliability and reliable life are under the exponential distribution, Weibull distribution, and normal distribution [6]. Two years later, Chen et al. [7] proposed the optimal lower confidence limit method for average life, reliability, and reliable life using the product under several different life distribution conditions. Based on the previously mentioned results, Sun and Chen [8] used the confidence limit method for reliability assessment studies under the condition that the product lifetime obeys the Weibull distribution and the lognormal distribution.

The confidence limit evaluation method is an efficient way to deal with the reliability evaluation of zero-failure data. It is often used for reliability estimation in the case of 
zero-failure data. Although the related research on reliability evaluation methods based on zero-failure data started late, the development in the past decade has also made some progress. Fu and Zhang [9] proposed a zero-failure data reliability analysis method with a known lower bound of shape parameters under the condition that the product life is subject to the Weibull distribution and gave a concrete expression of the one-side confidence lower limit of service life and reliability. After Monte Carlo simulation and simulation analysis, it is verified that the proposed method can make full use of zero-failure data and can accumulate the nonfailure data whose product life is subject to the distribution and increase the credibility of the information volume to improve the results. Jiang et al. $[3,10]$ extended the research on the product failure rate estimation method and proposed the least squares estimation and Bayesian estimation of the failure rate under the different life distributions. The estimation method combined with an example verifies the effectiveness and robustness of the method. Chambal and Bertkeats [11] proposed to use triangle distribution instead of Weibull distribution to describe the failure rate of components. The zero-failure data confidence limit evaluation method is widely used due to its strong generalization ability. Kayis [12] took the parameters related to product reliability as random variables and estimated the reliability under the unilateral confidence limit by using the parameters of different confidence intervals. Based on a class of nonlinear tunnel diode circuits with parameter perturbation, Chang et al. established the Takagi-Sugeno fuzzy model for the uncertainty of parameters, which effectively achieved the purpose of failure filtering errors [13]. In the process of expression of reliability uncertainty, the nonlinear functions are identified via neural networks can be effectively described, and the neural networks-based switched observer is constructed to approximate all unmeasurable states [14]. The uncertainty research in the reliability research process often used the neural network method to estimate the function, which would effectively solve the problem of considering the saturation nonlinearity [15]. Han [16] and Jiang and Jiang [17] put forward the optimal confidence limit method and applied it in different life distribution types and the single confidence limit of reliability was obtained according to the definite reliability analysis requirements.

Based on the existing research, a confidence limit method set (CLMS) is established in the Weibull distribution for reliability estimation of zero-failure data. The method set includes the unilateral confidence limit method and optimal confidence limit method, so that almost all existing grouping types of zerofailure data can be quickly evaluated, and multiple methods can be used in parallel to deal with the same problem. In this study, the grouping types of zero-failure data involved in the research object are combed in detail. Based on different zero-failure data grouping forms and related usage conditions, the confidence limit analysis methods used are different. The method set can be used to quickly and accurately select the appropriate confidence limit evaluation method for reliability estimation. The effectiveness and high efficiency of the CLMS combined with numerical simulation examples have been verified, and the possibility of analyzing multiple groups of zero-failure data with a confidence limit method suitable for processing single group of zero-failure data is expanded. Finally, in order to verify the effect of this method set in practical engineering, the actual case analysis of the single group of zero-failure data of rolling bearings and the multiple groups of zero-failure data of torque motors was conducted. This method set will facilitate future research, so that we can quickly find a way to solve the problem when encountering similar problems.

In this paper, the reliability estimation for zero-failure data based on confidence limit analysis method for industrial products is discussed. First of all, we introduce the characteristics of the Weibull distribution model and the physical properties of the product life distribution that each parameter can reflect. Then, we elaborate on the reasons and classification of zero-failure data. Third, in the section about the application and conditions of use of the confidence limit assessment method, the specific technical route and application scenarios of the method are described in detail. Finally, we use the representative rolling bearing and torque motor examples of industrial products to carry out verification research with the method proposed in this paper to further verify the effectiveness of the CLMS proposed in this paper.

\section{Weibull Distribution}

The Weibull distribution is widely used in the research of reliability assessment and product life prediction due to its own properties and good applicability in industrial product life statistics [18]. Most electronic, mechanical, and electrical products (such as bearings, generators, hydraulic pumps, and materials) are subjected to this distribution [19]. The probability density function (PDF) of the three-parameter Weibull distribution is defined as

$$
f(t)=\frac{\beta}{\vartheta}\left(\frac{t-\gamma}{\vartheta}\right)^{\beta-1} \exp \left[-\left(\frac{t-\gamma}{\vartheta}\right)^{\beta}\right], \quad t \geq \gamma,
$$

where $\beta$ indicates shape parameters, $\vartheta$ indicates scale parameters, and $\gamma$ indicates position parameters.

When the value of position parameter $\gamma$ is 0 , the threeparameter Weibull distribution degenerates into two-parameter Weibull distribution. After nearly 80 years of research and application, the statistical analysis of a large number of engineering test data samples proves that Weibull distribution model plays an important role in the research of product life distribution type and reliability assessment [20]. The cumulative distribution function (CDF) is defined as

$$
F(t)=P(T \leq t)=1-\exp \left[-\left(\frac{t-\gamma}{\vartheta}\right)^{\beta}\right], \quad t \geq \gamma,
$$

where $T \sim W(\beta, \eta, \gamma)$, for $R(t)=1-F(t)$, and the reliability function is

$$
R(t)=\exp \left[-\left(\frac{t-\gamma}{\vartheta}\right)^{\beta}\right], \quad t \geq \gamma
$$

The failure rate of industrial products such as bearings and motors usually has the characteristics of gradually 
increasing and decreasing with time. With regard to the geometric meaning of the position parameter, the position parameter $\gamma$ can determine the starting position of the distribution curve in the coordinate system, and its value change causes the curve to move in parallel on the coordinates. With regard to the physical meaning of the position parameter, when the position parameter $\gamma<0$, this indicates that the product fails before use; when the position parameter $\gamma>0$, this indicates that the product starts to fail, and when the mission time is greater than $\gamma$, the probability of failure before this time is 0 . Therefore, the value of the position parameter in the process of actual engineering application is also called the minimum life or the safe life. The change of the position parameter causes the probability density function of the distribution to change as shown in Figure 1.

\section{The Grouping Type of Zero-Failure Data}

Currently, there are mainly two kinds of zero-failure data grouping type, one is test data of a single group, and the other is test data of multiple groups [21]. Among them, the multipacket-type zero-failure data can be further subdivided into pretest grouping and posttest grouping. The censoring time is generally set in advance in the reliability life test, which has great subjectivity and dependence on expert experience. The preset value of the censoring time is further divided into regular and random settings. The grouping type of zero-failure data is shown in Figure 2.

3.1. Single Group of Zero-Failure Data. When $n$ samples participate in the censoring test, it ends at time $t$, and all samples do not fail. At this time, a set of zero-failure data is obtained, which can be expressed as $Z=(n, t)$. The value of time $t$ can be set before or after the censored test.

3.2. Multiple Groups of Zero-Failure Data. A batch of samples is randomly selected from the products of the study subjects for life test, and the sample capacity is represented by $N$. The batch of samples is divided into $m$ groups, and the number of samples included in each group is represented by $n_{i}$, and the corresponding end time is $t_{1}, t_{2}, \ldots, t_{m}$ $\left(t_{1}<t_{2}<\cdots<t_{m}\right)$. At this time, the corresponding zerofailure data obtained according to the timing censored test can be expressed as $Z=\left(n_{i}, t_{i}\right)$. The value of $m$ can be set before or after the start of the test, and the setting of the parameter $n_{i}$ can be random or a regular value.

After the previously mentioned analysis, it is found that the single-group type of zero-failure data belongs to the special case of the multiple groups' type.

\section{The Confidence Limit Assessment Method}

\subsection{Unilateral Confidence Limit Assessment Method}

4.1.1. The Assessment for Single Group of Zero-Failure Data. If the premise of the life of the product obeying the twoparameter Weibull distribution after the statistical analysis of the previous empirical data is established, it is often difficult to

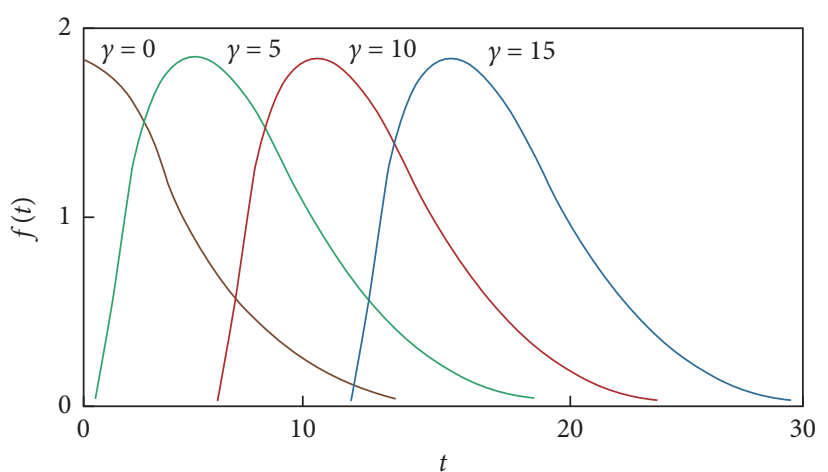

FIGURE 1: Probability density function curves with different position parameters.

directly estimate the shape parameters in the life distribution by using the zero-failure data. However, when the range of the shape parameters can be determined, the theoretical logic deduction can be used to derive the unilateral confidence limit of the reliability parameter of the samples.

There is such a set of zero-failure data, the sample size is $n$, and the censoring time is $t_{0}$. The value of time $t_{0}$ can be set before or after the censored test. According to the exponential distribution characteristics [9], the reliability $R_{l}$ of the sample under the condition that the confidence level is $1-\alpha$ can be expressed as

$$
R_{l}(t)=\exp \left(\frac{t \ln (\alpha)}{N}\right),
$$

where $t$ indicates mission time, and $N=(n+1) \mathrm{t}_{0}$. Using formula (4), the lower confidence interval of the mean time between failures (MTBF) in the case of a single group zerofailure data can be obtained as

$$
\theta_{l}=-\frac{(n+1) t_{0}}{\ln \alpha}
$$

If the shape parameter of Weibull distribution is known, let $X=t^{\beta}, \theta=\eta^{\beta}$, and then it can be considered that $X$ obeys the exponential distribution. After transformation, the Weibull distribution is transformed into an exponential distribution, and the following can be obtained as

$$
\theta_{u 1}=\eta_{u 1}^{\beta}=-\frac{(n+1) t_{0}^{\beta}}{\ln \alpha}
$$

The reliability unilateral confidence limit under the Weibull distribution is obtained by (6), and the $R_{u 1}$ of under the condition that the confidence level is $1-\alpha$ can be expressed as

$$
R_{u 1}(t)=\exp \left[\frac{\ln \alpha}{n+1}\left(\frac{t}{t_{0}}\right)^{\beta}\right],
$$

where $t$ indicates mission time, $\beta$ indicates shape parameters, and $\vartheta$ indicates scale parameters.

At the same time, under the condition that the confidence level is $1-\alpha$, it is also possible to obtain the unilateral confidence limit of the reliability when the lower limit of the 


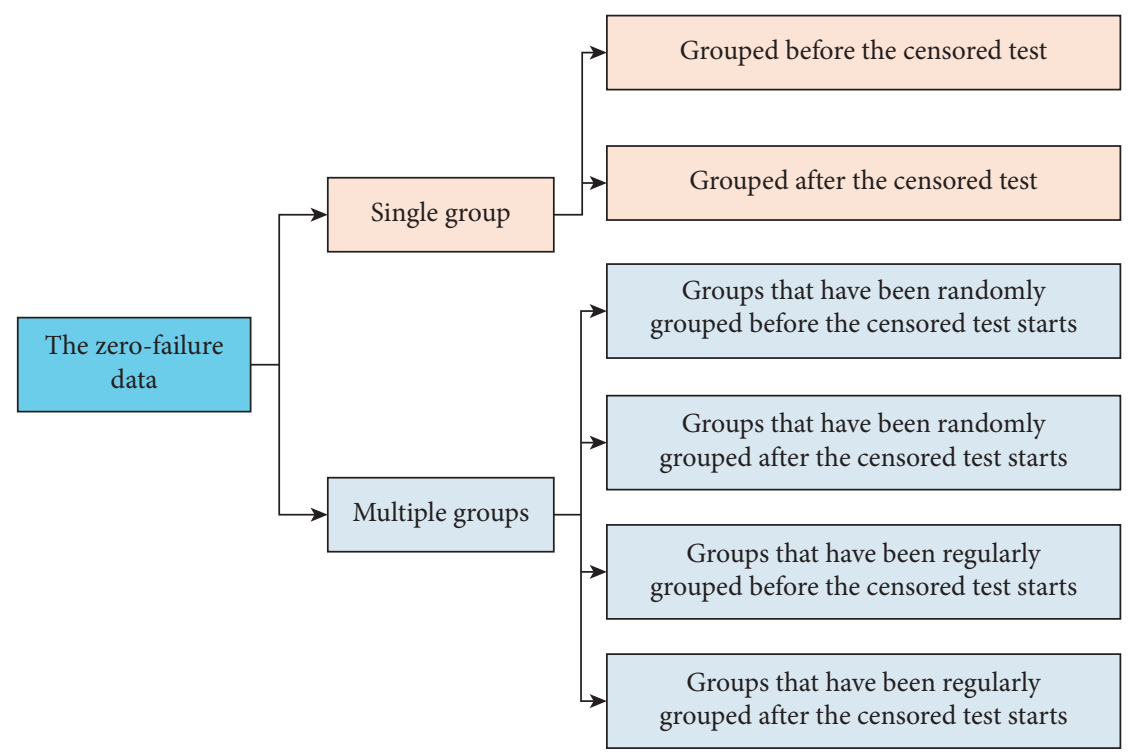

FIgURE 2: The grouping type chart of zero-failure data.

value range of the shape parameter in the Weibull distribution is known. The unilateral confidence limit of the reliability $R_{u 1}^{\prime}$ can be expressed as

$$
R_{u 1}^{\prime}(t)=\exp \left[\frac{\ln \alpha}{n+1}\left(\frac{t}{t_{0}}\right)^{\beta_{0}}\right], \quad(t>0),
$$

where $t$ indicates the mission time and $\beta_{0}$ indicates the lower limit of the shape parameter.

4.1.2. The Assessment for Multiple Groups of Zero-Failure Data. There is such a batch of multiple groups of zerofailure data, and the sample size is $n$. The number of groups is $m$, each group contains the sample number $n_{i},(i=1,2, \ldots, m)$, and the corresponding censoring time is $t_{1}, t_{2}, \ldots, t_{\mathrm{m}}$ and the condition is $t_{1}<t_{2}<\cdots<t_{m}$. In this case, zero-failure data can be represented as $Z=\left(t_{i}, n_{i}\right)$, and the reliability $R_{u 2}$ of products under the condition that the confidence level is $1-\alpha$ can be expressed as [10]

$$
R_{u 2}(t)=\exp \left[\frac{\left(t^{\beta} \ln \alpha\right)}{\sum_{i=1}^{m} n_{i} t_{i}^{\beta}}\right], \quad t>0 .
$$

The derivation process of this reliability evaluation expression is also combined with the idea of exponential distribution parameter estimation, and the specific process is omitted here.

\subsection{Optimal Confidence Limit Assessment Method}

4.2.1. The Assessment for Single Group of Zero-Failure Data. According to the characteristics of zero-failure data of the single group, there is such a set of zero-failure data, the sample size is $n$, and the censoring time is $t_{0}$. The optimal confidence limit analysis method for single group of zero- failure data is a simplified result of multiple groups of forms.

Under the condition that the shape $\operatorname{parameter} \beta$ in the two-parameter Weibull distribution is unknown [7], the reliability $R_{o 1}$ under the condition that the confidence level is $1-\alpha$ can be expressed as

$$
R_{o 1}(t)= \begin{cases}0, & t>t_{0}, \\ \alpha^{1 / n}, & 0<t \leq t_{0},\end{cases}
$$

where $t$ indicates the mission time.

If the shape parameter $\beta$ has a value range of $\left[\beta_{1}, \beta_{2}\right]$, the reliability $R_{o 1}^{\prime}$ under the condition that the confidence level is $1-\alpha$ can be expressed as

$$
R_{o 1}^{\prime}(t)= \begin{cases}\alpha^{1 /\left[n\left(t_{0} / t\right)^{\beta_{1}}\right]}, & 0<t<t_{0}, \\ \alpha^{1 /\left[n\left(t_{0} / t\right)^{\beta 2}\right]}, & t \geq t_{0},\end{cases}
$$

where $t$ indicates the mission time.

The range of shape parameters in the two-parameter Weibull distribution often comes from the statistical results of product life data in engineering practice. The optimal confidence limit analysis method under zero-failure data is more reliable because of the introduction of product life distribution parameter information.

\subsubsection{The Assessment for Multiple Groups of Zero-Failure} Data. According to the characteristics of zero-failure data of the multiple groups, the number of groups is $m$, each group contains the sample number $n_{i},(i=1,2, \ldots, m)$, and the corresponding censored time is $t_{1}, t_{2}, \ldots, t_{\mathrm{m}}$ and the condition ist $t_{1}<t_{2}<\cdots<t_{m}$. In this case, zero-failure data can be represented as $Z=\left(\mathrm{t}_{i}, n_{i}\right)$.

According to [7], when the confidence level is $1-\alpha$, the lower confidence limit $R_{o 2}$ can be expressed as 


$$
R_{o 2}(t)=\inf \left\{\exp \left[-\left(\frac{t}{\vartheta}\right)^{\beta}\right]\right\}: \prod_{i=1}^{m} \exp \left[-\left(\frac{t_{i}}{\vartheta}\right)^{\beta}>\alpha, \eta>0, \beta>0\right]=e^{-a}
$$

where $\quad a=\sup \left[(t / \vartheta)^{\beta}: \sum_{i=1}^{m} t_{i}^{\beta} /-\ln \alpha<\vartheta^{\beta}, \vartheta>0, \beta>0\right]=$ $-\ln \alpha / \inf \{f(\beta): \beta>0\}$; at this time there is the equation$f(\beta)=\sum_{i=1}^{m}\left(t_{i} / t\right)^{\beta}$. We can get the inequality $f^{\prime}(\beta) \geq 0$ when the condition is $t \leq\left(\prod_{i=1}^{m} t_{i}\right)^{1 / m}$. So, $\inf \{f(\beta): \beta>0\}=\lim _{\beta \longrightarrow 0} f(\beta)=m$. The lower confidence limit for reliability at this mission time is

$$
R_{o 2}(t)=\alpha^{1 / m}
$$

The inequality is $f^{\prime}(\beta) \leq 0$ when the condition is $t \geq t_{(m)}$, where, $\mathrm{t}_{(m)}=\max \left(t_{1}, t_{2}, \ldots, t_{m}\right)$; therefore,

$$
\inf \{f(\beta): \beta>0\}=\lim _{\beta \rightarrow \infty} f(\beta)= \begin{cases}0, & \text { when } t>t_{(m)}, \\ P, & \text { when } t=t_{(m)},\end{cases}
$$

where the parameter $P$ is an element in the set $\left\{i: 1 \leq i \leq m, t_{i}=t_{(m)}\right\}$, and the lower confidence limit for reliability at this time is

$$
R_{o 2}(t)= \begin{cases}0, & \text { when } t>t_{(m)} \\ \alpha^{1 / P}, & \text { when } t=t_{(m)}\end{cases}
$$

The parameter $\beta^{*}$ is the only solution of the equation $\sum_{i=1}^{m}\left(t_{i} / t\right)^{\beta} \ln \left(t_{i} / t\right)=0$, when $\left(\prod_{i=1}^{m} t_{i}\right)^{1 / m}<t<t_{(m)}$, and theninf $\{f(\beta): \beta>0\}=\lim f\left(\beta^{*}\right)$. The lower confidence limit for reliability at this time is $R_{o 2}=\alpha^{1 / f\left(\beta^{*}\right)}$. Under the condition that the shape parameter $\beta$ in the two-parameter Weibull distribution is unknown, the reliability $R_{o 2}$ under the condition that the confidence level is $1-\alpha$ can be expressed as

$$
R_{o 2}(t)= \begin{cases}0, & t>t_{(m)}, \\ \alpha^{1 / P}, & t=t_{(m)}, \\ \alpha^{1 / f\left(\beta^{*}\right)}, & c<t<t_{(m)}, \\ \alpha^{1 / m}, & 0<t \leq c,\end{cases}
$$

where $t$ indicates the mission time; $c=\left(\prod_{i=1}^{m} t_{i}\right)^{1 / m}$.

If the shape parameter $\beta$ has a value range of $\left[\beta_{1}, \beta_{2}\right]$, the reliability $R_{o 2}^{\prime}$ under the condition that the confidence level is $1-\alpha$ can be expressed as follows:

$$
R_{o 2}^{\prime}(t)= \begin{cases}\alpha^{1 / f\left(\beta_{1}\right)}, & 0<t \leq c, \\ \alpha^{1 / f\left(\beta_{2}\right)}, & t \geq t_{(m)}, \\ \alpha^{1 / f\left(\beta_{1}\right)}, & c<t<t_{(m)}, \beta^{*} \leq \beta_{1}, \\ \alpha^{1 / f\left(\beta_{2}\right)}, & c<t<t_{(m)}, \beta^{*} \geq \beta_{2}, \\ \alpha^{1 / f\left(\beta^{*}\right)}, & c<t<t_{(m)}, \beta_{1} \leq \beta^{*} \leq \beta_{2},\end{cases}
$$

where $t$ indicates the mission time $t_{(m)}=\max \left(t_{1}, t_{2}, \ldots, t_{m}\right)$, the parameter $\beta^{*}$ is the only solution of the equation $\sum_{i=1}^{m}\left(t_{i} / t\right)^{\beta} \ln \left(t_{i} / t\right)=0$, and $f(\beta)=\sum_{i=1}^{m}\left(t_{i} / t\right)^{\beta}, c=\left(\prod_{i=}\right.$ $\left.1^{m} t_{i}\right)^{1 / m}$.

\section{Establishing the Confidence Limit Method Set}

In the Weibull distribution, all the current commonly used confidence limit analysis methods are collected into one framework to establish a method set, and we name it the confidence limit method set (CLMS). The purpose of the method set is to meet the random engineering characteristics of practical engineering cases in the application process of reliability evaluation based on zero-failure data. In order to use the single group optimal confidence limit method to process multiple groups of zero-failure data, a method to extend the application of optimal confidence limits is proposed in this method set. The basic structure of the CLMS is shown in Figure 3.

It can be seen from the schematic diagram that one side of the method set is the existing confidence limit analysis method, and the other side is the form of the zero-failure data case in actual engineering. The direction indicated by the arrow in the middle represents which cases the method can be used to evaluate. This makes it possible to apply multiple methods at the same time to solve a problem, and then select the optimal reliability evaluation result. The establishment of this method set belongs to a kind of ideological practice. With the deepening and development of research in this field, this method set can continue to expand to more methods and more types of life distribution. It can be seen from the source of zero-failure data that this type of life data does not contain the dispersive information of the actual life of the product, so it is difficult to estimate accurate shape parameters based on the zero-failure data. However, in the actual engineering application process, through past experience and actual product life statistics, we can often get the value range of the shape parameter in the Weibull distribution. It is feasible to conduct reliability assessment by introducing empirical life distribution parameter information into the confidence limit evaluation method of zerofailure data.

\section{Simulation Verification and Discussion}

According to previous research experience and results, it is assumed that the bearing life of the research object follows the three-parameters Weibull distribution. Yang [22] analyzed the fatigue life test data of 135 groups, 6 types, 52 models, and a total of 2031 sets of bearings from the National Bearing Quality Monitoring Center of Luoyang Bearing Research Institute and various bearing companies for a long time and obtained the shape parameter of ball bearings. Possible values are 1.5 and rolling bearings are 1.7. Therefore, under the condition that the bearing products are subject to Weibull distribution, assuming that the shape parameter value is 2.0 , it can meet the analysis requirements of conventional reliability problems. 


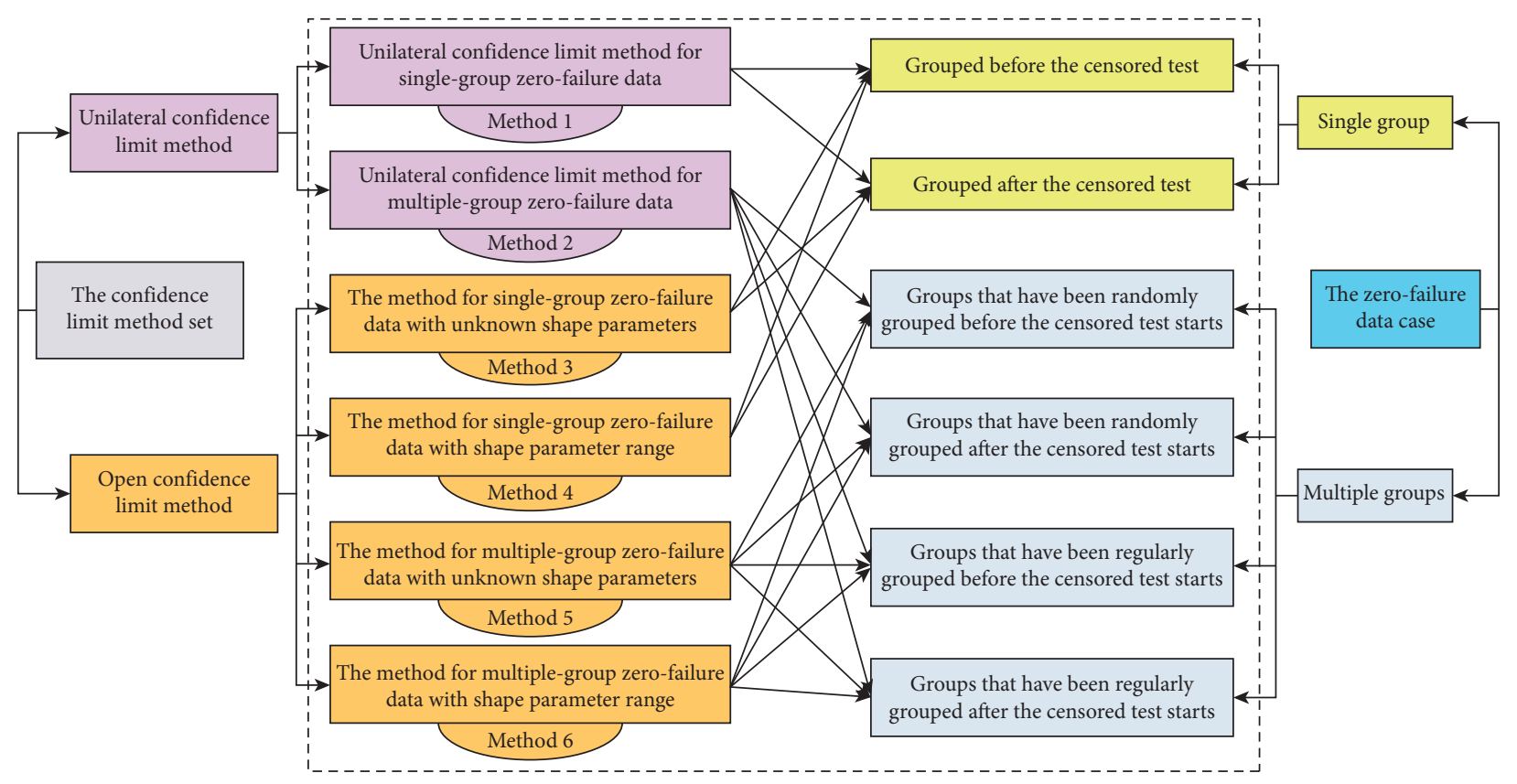

Figure 3: The CMLS for reliability evaluation based on zero-failure data.

The zero-failure data of the numerical simulation of bearings are listed in Table 1. The data are generated based on the method of generating zero-failure data samples [23] and the results of previous statistical studies on the life of rolling bearings of this type. Then, a set of pseudorandom numbers is generated under the conditions of specifying shape parameters and scale parameters based on the method of generating zero-failure data samples. Finally, a set of random numbers that follow the Weibull distribution (the number of random numbers should be much greater than the number in the bearing reliability test set) is generated by using the Monte Carlo method. The number of random numbers generated is 600 . The values of relevant parameters in the zero-failure data of a group of bearings generated by simulation include the following: shape parameter is $\beta=2.0$, scale parameter is $\vartheta=2000$, and position parameter is $\gamma=1000$. The mission time $t$ in the two-parameter Weibull distribution is equivalent to the $t-\gamma$ in the three-parameter Weibull distribution.

There are 55 samples of zero-failure data for this group of bearings, as shown in Table 1. In order to use the single group optimal confidence limit method to process multiple groups of zero-failure data, a method to extend the application of optimal confidence limits is proposed in this part. The feasible solution is to average-equivalently process the total time $T=\sum_{i=1}^{m} n_{i} t_{i}$, and then the single censoring time $t_{0}=\sum_{i=1}^{m} n_{i} t_{i} / n$, where $\mathrm{n}=55$. Using the optimal confidence limit method for single group of zero-failure data to calculate the reliability estimation results of this simulation example is shown in Figure 4; the confidence level is $1-\alpha=0.95$. It can be known that the life of the batch of bearings is subject to Weibull distribution, and the shape parameter values range from 1.8 to 2.3 [23]. Based on the current assumptions and the CMLS, we can use three methods to estimate the reliability of this group of bearings. The following analysis content is the lower confidence limit of product reliability in three cases, where the shape parameter is unknown (Method 3 ) and the shape parameter range is known (Method 1 and Method 4).

Although using the data set can quickly find the evaluation method, it can be seen from the evaluation results in Figure 4 that the evaluation effect after simplified processing is not ideal. The reason why the curve of the reliability estimate value in this figure is not smooth is that the value points of the reliability estimate are too scattered, but this does not affect the comparative analysis between different evaluation methods. Compared with the true value of the reliability change trend, the reliability estimates of the three methods are either too high or too low. The estimate of the lower limit of reliability in Method 3 is 0 under the condition that the task time is greater than the maximum censoring time $t_{0}$, and it has nothing to do with the confidence level. Therefore, Method 3 has certain limitations. According to the analysis of the results of this simulation example, the reliability estimation has always been on the high side by Methods 1 and 4. This will lead to underestimation of the probability of bearing risk in actual engineering applications.

Based on the data in Table 1, the lower confidence limit of product reliability is analyzed in the case where the shape parameter is unknown and the shape parameter range is known. In the following analysis, the confidence level parameter is $1-\alpha=0.95$, and the shape parameter assumption is still unknown or known to have a range of 1.8-2.3. After calculation, the parameter of critical time node is $c=2375.8 \mathrm{~h}$. Based on the current assumptions and CMLS, three methods are used to estimate the reliability through this set of zero-failure data samples. The following analysis content is the lower confidence limit of product reliability in two cases, where the shape parameter is unknown (Method 5 ) and the shape parameter range is known (Method 2 and 
TABLE 1: Zero-failure data sample of bearing based on three-parameter Weibull distribution.

\begin{tabular}{lccc}
\hline Censoring time/h & The number of samples $n_{i}$ & Censoring time $(\mathrm{h})$ & The number of samples $n_{i}$ \\
\hline 1030 & 10 & 2632 & 5 \\
1617 & 9 & 2871 & 4 \\
1915 & 8 & 3195 & 3 \\
2180 & 7 & 3581 & 2 \\
2379 & 6 & 4006 & 1 \\
& & Total number & 55 \\
\hline
\end{tabular}

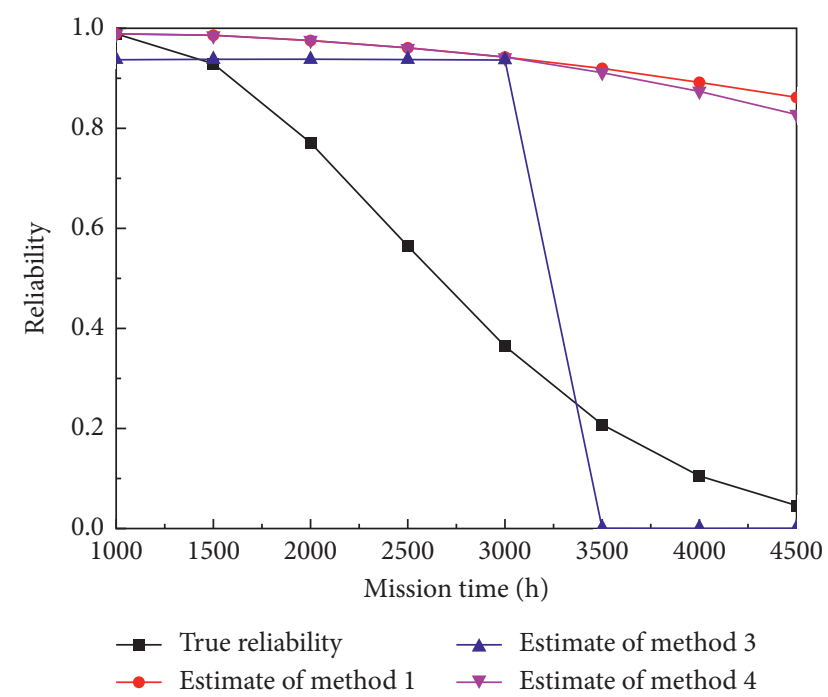

Figure 4: Reliability lower confidence limit estimate for single set of zero-failure data.

Method 6), and the results of the optimal confidence limit evaluation are shown in Figure 5.

From the results in Figure 5, the type of zero-failure data grouping has a direct impact on the reliability evaluation during the application of the optimal confidence limit analysis method. The reliability estimated by the unilateral confidence limit assessment method (Method 2) is significantly higher than the true value. The reliability estimates obtained by the two methods (Methods 5 and 6 are closest to the true value when the mission time is near critical time node $c$ ). The estimates of the lower confidence limits of reliability obtained by the two methods are conservative when the task time is less than 2000 hours. However, the estimates of the lower confidence limits of the reliability obtained by the two methods are biased when the task time is greater than 2000 hours. Although Method 6 considers more time stages to estimate the reliability, Method 5 is generally better. The optimal confidence limit evaluation method plays a role in dealing with rare events in failure analysis (reliability evaluation based on zero-failure data) and the estimates near critical time nodes are still very close to the true value of reliability, which can provide a reference for practical engineering applications.

\section{Case Study}

7.1. Single Group of Zero-Failure Data for Bearings. According to the rolling bearings used at the joints of a certain type of robot in the laboratory environment, we get such a set of zero-failure data. The sample size of this set of zero-failure data is 3 , the censoring time is 1200 hours, and the zero-failure data of the bearing is expressed as $Z=\left(t_{0}, n\right)=(1200,3)$. The photograph of the rolling bearing before it is not installed at the joint is shown in Figure 6. Therefore, for simplicity and without loss of generalization ability, it is assumed that the life of this group of bearings follows the two-parameter Weibull distribution, and the shape parameter ranges from 1.5 to 2.3. In the following analysis, the confidence level parameter is $1-\alpha=0.95$.

Based on the current assumptions and CMLS, we can use two methods (Methods 1 and 4) to estimate the reliability of this group of bearings. The reliability evaluation results of the bearings are shown in Figure 7.

Combined with the results of the numerical simulation study, it can be known that the reliability estimated by the optimal confidence limit method is closer to the true value, and it can be seen that Method 4 estimates the reliability of the bearing better. At the same time, it can also be found that the effects of the two methods when processing a single set of zero-failure data are very close. In order to analyze the influence of the sample size on the evaluation results; on the basis of this example, it is assumed that the sample size increases, and the results of the reliability evaluation according to the optimal confidence limit analysis method are shown in Figure 8.

From the results in Figure 8, it can be seen that the larger the sample size, the higher the final reliability estimate, and the change rate of the reliability estimate gradually decreases as the task time increases. Such conclusions are in accordance with the objective law of reliability evaluation through zero-failure data.

7.2. Multiple Groups of Zero-Failure Data for Torque Motor. The zero-failure data of this study came from the field operation data of the torque motors. Recording the time data of a product from start to failure (or failure-free) is an important data source for reliability evaluation. The reliability data of the actual work scene is extremely valuable. It reflects the operation of the product in the actual environment and maintenance conditions and is more representative of the product characteristics than the simulated data in the laboratory environment. The figure of the torque motor sample is obtained from [24] as shown in Figure 9.

In order to analyze the reliability of the torque motors, the operation records of 52 torque motors used in CNC machine tools are reviewed. After analysis and arrangement, 


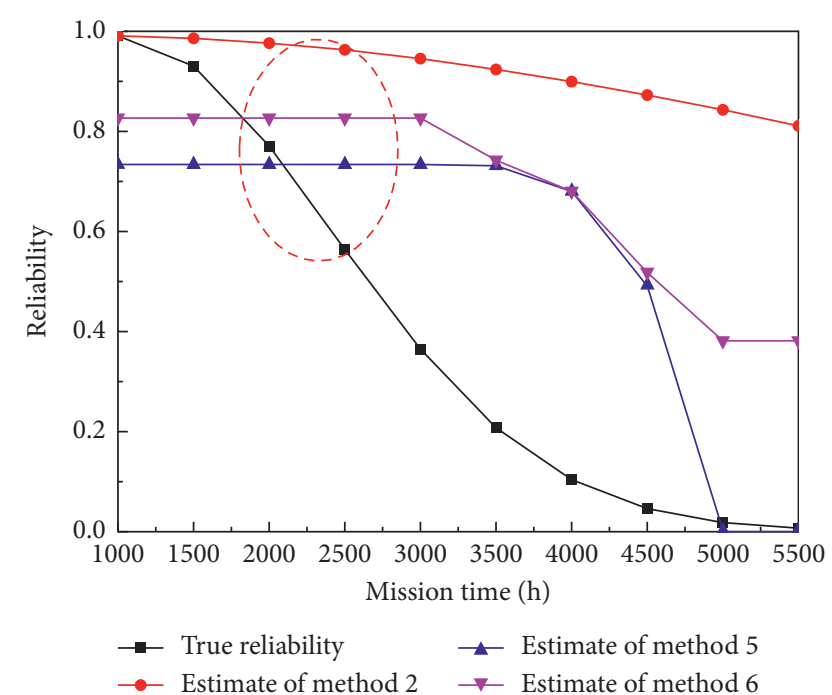

FIGURE 5: Reliability lower confidence limit estimate for multiple groups of zero-failure data.

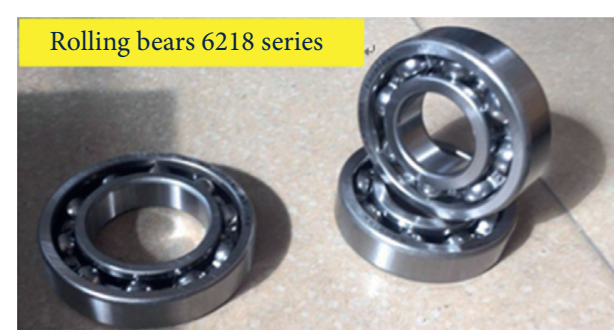

Figure 6: The rolling bearing sample.

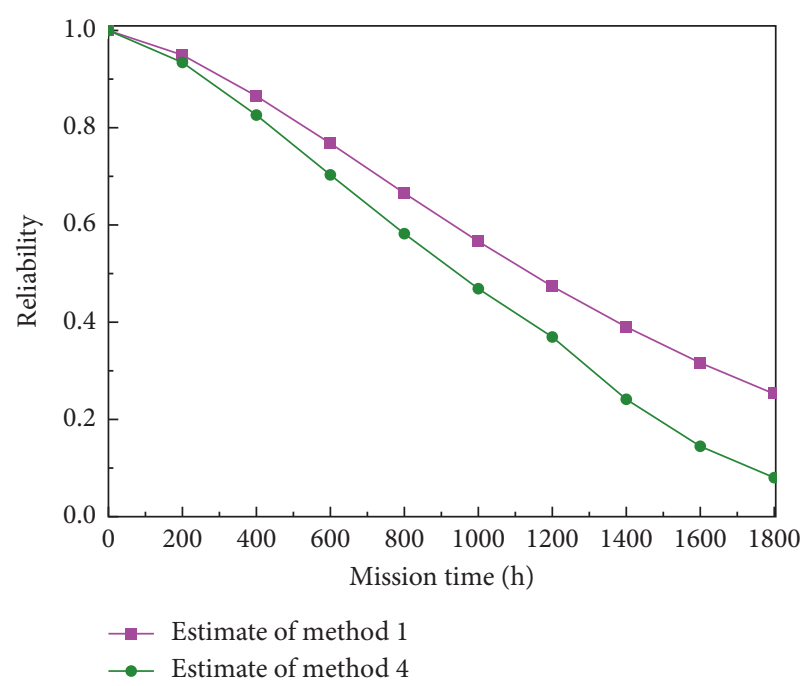

FIgURE 7: The comparison of reliability estimation results of two methods.

it is found that 52 torque motors have no fault in the mission time. As the starting time of the type-I censoring experiment, the time of the last recording of the motors in the $\mathrm{CNC}$

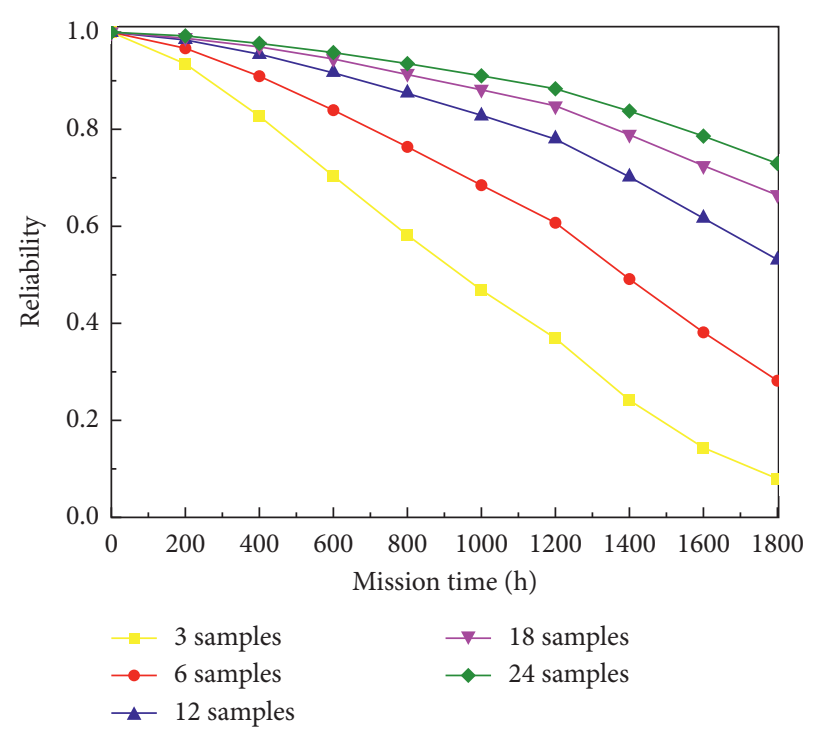

FIGURE 8: Changes in reliability estimates are based on different sample size.

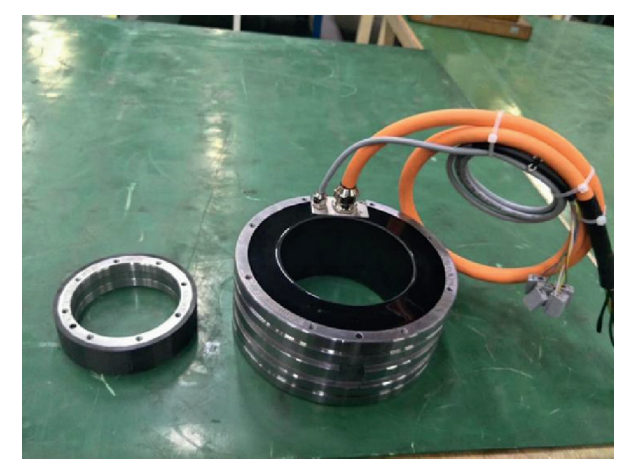

Figure 9: A torque motor sample of this set [24].

machine tool is used as the censoring time of the experiment, and then the running time $t_{i}$ corresponding to the torque motors is given. The sample data of the torque motor is obtained from [24] as shown in Table 2.

According to the previous research results [21], the batch of torque motors follows the two-parameter Weibull distribution, and the shape parameter range is between 1.5 and 1.8. Based on the previously mentioned analysis results and the confidence limit method set, for the reliability evaluation of the torque motor example, it is advisable to adopt multiple sets of zero-failure data evaluation methods in the unilateral confidence limit method and the optimal confidence limit method. Since the estimated value of the shape parameter is within a range, the interval estimation result of the reliability obtained by the unilateral confidence limit analysis method is evaluated. Although this example can refer to the range of the shape parameter interval, according to the results of numerical simulation analysis, Method 3 should be used when using the optimal confidence limit analysis. In the following analysis, the confidence level parameter is $1-\alpha=0.95$. Based on the current assumptions and the CMLS, we can use two methods 
TABLE 2: The zero-failure data sample of torque motor [24].

\begin{tabular}{lccc}
\hline Censoring time/h & The number of samples $n_{i}$ & Censoring time/h & The number of samples $n_{i}$ \\
\hline 256 & 2 & 2960 & 2 \\
720 & 2 & 3600 & 2 \\
960 & 2 & 4240 & 4 \\
1200 & 2 & 4320 & 10 \\
1360 & 2 & 4960 & 2 \\
1440 & 6 & 5440 & 3 \\
2400 & 2 & 5520 & 1 \\
2560 & 2 & 5760 & 2 \\
2640 & 2 & 7200 & 4 \\
& & Total number & 52 \\
\hline
\end{tabular}

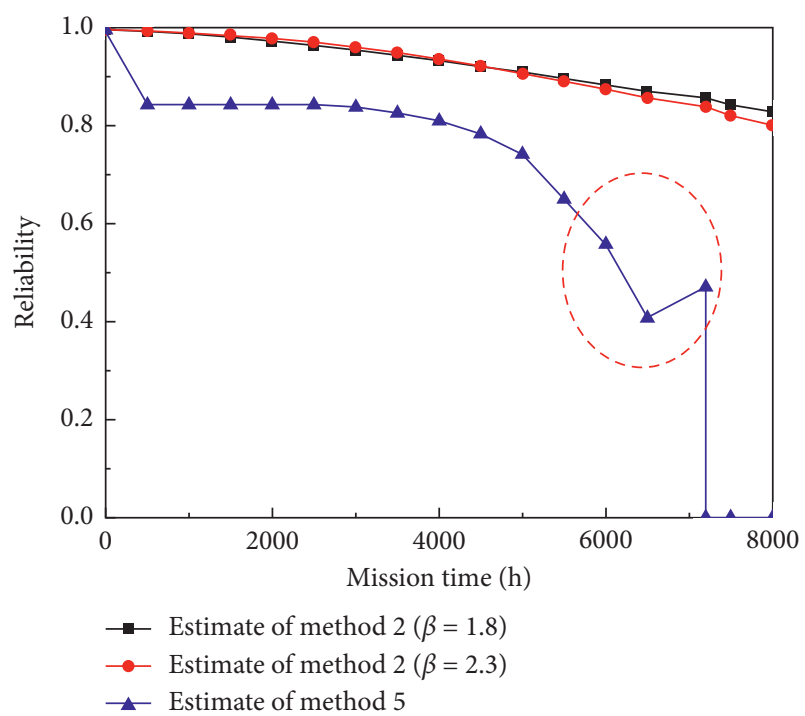

Figure 10: The comparison of reliability estimation results of two methods.

(Methods 2 and 5) to estimate the reliability of this group of torque motors.

As can be seen from Figure 10, the reliability evaluation results using Method 2 are not sensitive to the shape parameter values; that is to say, when the shape parameter values change a little, the reliability estimate value increases and the task time increase is almost negligible. According to the analysis of the evaluation results of Method 5, if the life distribution assumption of the torque motor is accurate, then the estimated value of the reliability of the accessory $\mathrm{C}$ at the critical time is about 0.85 . It can be seen that the estimation results of Method 2 are biased. The result of the reliability estimation value using Method 5 has a fluctuation phenomenon when the task time is the maximum censoring time. This is because the expression of the reliability estimation value is $R_{o 2}(t)=\alpha^{1 / p}$. The relationship between the estimated reliability at this moment and the change in sample size is shown in Figure 11.

It can be seen that the number of samples corresponding to the maximum censoring time directly affects the size of

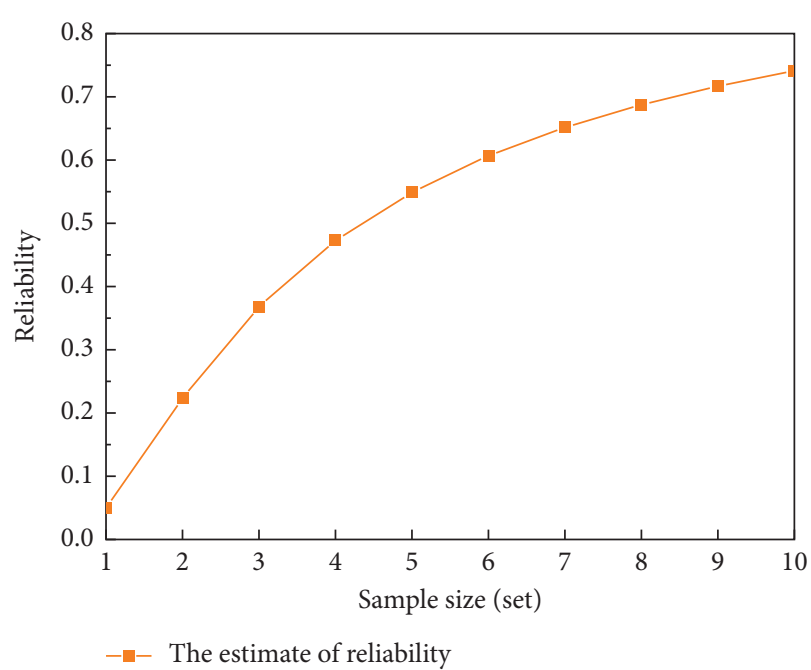

Figure 11: The increase in the number of samples leads to changes in reliability estimates value.

the reliability estimate under the task time. In this case study, the occurrence of the final reliability estimate value fluctuating at the maximum truncation time point is the phenomenon caused by this reason.

\section{Conclusion}

This paper gives a unilateral confidence limit analysis method and an optimal confidence limit analysis method for product life that follow the Weibull distribution. According to past engineering experience, the shape parameter value information of the product life distribution is introduced to obtain a more accurate evaluation effect. By establishing a set of confidence limit methods for analyzing zero-failure data, a quick search method is realized, and the optimal reliability estimation result is selected using the comparative analysis results. The currently established method set is only applicable to the evaluation of various types of zero-failure data under the condition of Weibull distribution. Combining numerical simulation examples and case studies, the following conclusions are obtained.

(1) Through the method set established in this paper, the reliability evaluation of zero-failure data can be used to quickly select the methods that need to be adopted 
in the case of the Weibull distribution, and the optimal evaluation results are selected through lateral comparison analysis.

(2) Through the analysis of examples and numerical simulation examples, it can be seen that the reliability estimate obtained by the confidence limit analysis method is closer to the real situation when the task time is shorter.

(3) The shape parameter of the life distribution of the research object is a crucial factor, which directly affects the accuracy of the reliability estimation under the condition of Weibull distribution.

In the future, the research object industrial products will adopt a variety of different distribution types of confidence limit analysis methods to establish a more comprehensive method set, which will be able to meet a wider range of practical engineering applications.

\section{Data Availability}

The data used to support the findings of this study are included within the article.

\section{Conflicts of Interest}

The authors declare that they have no conflicts of interest.

\section{Acknowledgments}

This work was supported by the National Key Research and Development Program of China (grant no. 2018YFF0214704).

\section{References}

[1] P. Rani and G. S. Mahapatra, "A neuro-particle swarm optimization logistic model fitting algorithm for software reliability analysis," Proceedings of the Institution of Mechanical Engineers," Part O: Journal of Risk and Reliability, vol. 233, no. 6, 14 pages, 2019.

[2] C.-Y. Wei, X.-Q. Cai, B. Liu, T.-Y. Wang, and F. Gao, “A generic construction of quantum-oblivious-key-transferbased private query with ideal database security and zero failure," IEEE Transactions on Computers, vol. 67, no. 1, pp. 2-8, 2018.

[3] P. Jiang, Y. Y. Xing, X. Jia, and B. Guo, "Weibull failure probability estimation based on zero-failure data," Mathematical Problems in Engineering, vol. 2015, no. 3, 8 pages, Article ID 681232, 2015.

[4] H. F. Martz and R. A. Waller, "A bayesian zero-failure (BAZE) reliability demonstration testing procedure," Journal of Quality Technology, vol. 11, no. 3, pp. 128-138, 1979.

[5] C. L. Atwood and J. K. Vaurio, Zero Failure Data, Encyclopedia of Quantitative Risk Analysis and Assessment, John Wiley \& Sons, Inc, Hoboken, NY, USA, 2008.

[6] J. D. Chen, W. L. Sun, and B. X. Li, "The confidence limits for reliability parameters in the case of no failure data," in Proceedings of the Asian Conference on STA.Com, pp. 17-20, Beijing, China, 1993.
[7] J. D. Chen, W. L. Sun, and B. X. Li, "On the confidence limits in the case of no failure data," Acta Mathematicae Applicatae Sinica, vol. 18, no. 1, pp. 90-100, 1995.

[8] W. L. Sun and J. D. Chen, "Some new results on the confidence limits for reliability parameters in the case of no failures," Systems Science and Mathematical Sciences, vol. 01, pp. 70-81, 1999.

[9] H. M. Fu and Y. B. Zhang, "Method of reliability analysis for time truncated zero-failure data based on normal distribution," Journal of Aerospace Power, vol. 25, no. 2, pp. 384-387, 2010.

[10] H. B. Zhao and Y. M. Cheng, "Statistical analysis about zerofailure data using memory less property of exponential distribution," Chinese Journal of Applied Probability and Statistics, vol. 20, no. 1, pp. 59-65, 2004.

[11] S. Chambal and J. Bertkeats, "Evaluating complex system reliability using reliability block diagram simulation when little or no failure data are available," Quality Engineering, vol. 13, no. 2, pp. 169-177, 2000.

[12] S. A. Kayis, "Evaluation of confidence limit estimates of cluster analysis on molecular marker data," Journal of the Science of Food and Agriculture, vol. 92, no. 4, pp. 776-780, 2012.

[13] X. H. Chang, X. Jun, and J. H Park, "Estimation for a class of parameter-controlled tunnel diode circuits," IEEE Transactions on Systems, Man, and Cybernetics: Systems, vol. 50, no. 11, pp. 4697-4707, 2018.

[14] L. Ma, X. Huo, X. Zhao, and G. D. Zong, “Observer-based adaptive neural tracking control for output-constrained switched MIMO nonstrict-feedback nonlinear systems with unknown dead zone," Nonlinear Dynamics, vol. 99, no. 2, pp. 1019-1036, 2020.

[15] L. Ma, G. Zong, X. Zhao et al., "Observed-based adaptive finite-time tracking control for a class of nonstrict-feedback nonlinear systems with input saturation," Journal of the Franklin Institute, vol. 357, no. 16, pp. 11518-11544, 2019.

[16] M. Han, "Confidence limit of reliability parameters in the case of zero-failure data," Chinese Journal of Engineering Mathematics, vol. 21, no. 2, pp. 245-244, 2004.

[17] R. B. Jiang and D. Y. Jiang, "Fiducial lower confidence limits of reliability for a standby system based on zero-failure data," Zhejiang University.Science and Education, vol. 6, p. 629, 2010.

[18] D. A. Burger, R. Schall, J. T. Ferreira, and D.-G. Chen, "A robust Bayesian mixed effects approach for zero inflated and highly skewed longitudinal count data emanating from the zero inflated discrete weibull distribution," Statistics in Medicine, vol. 39, no. 9, p. 2, 2020.

[19] H. Pham and C.-D. Lai, "On recent generalizations of the weibull distribution," IEEE Transactions on Reliability, vol. 56, no. 3, pp. 454-458, 2007.

[20] A. Z. Djeddi, A. Hafaifa, A. Kouzou et al., "Exploration of reliability algorithms using modified weibull distribution: application on gas turbine," International Journal of System Assurance Engineering \& Management, vol. 8, no. 2, 10 pages, 2017.

[21] H. Li, L. Y. Xie, M. Li, J. Ren, B. Zhao, and S. Zhang, "Research on a new reliability assessment method for zero-failure data," Journal of Military Engineering, vol. 35, no. 01, pp. 1622-1631, 2019.

[22] X. W. Yang, Study on Three Parameters of Weibull Distribution for Fatigue Life of Rolling Bearings, Hefei University of Technology, Hefei, China, 2003.

[23] H. Y. Sun, Research on Bearing Reliability Based on No Failure Data, Northeastern University, Boston, MA, USA, 2014. 
[24] H. Li, L. Xie, M. Li, J. Ren, B. Zhao, and S. Zhang, "Reliability assessment of high-quality and long-life products based on zero-failure data," Quality and Reliability Engineering International, vol. 35, no. 1, pp. 470-482, 2019. 